\title{
Morbidity pattern and household cost of hospitalisation for non-communicable diseases (NCDs): a cross-sectional study at tertiary care level
}

\section{A Kasturiratne ${ }^{1}$, AR Wickremasinghe ${ }^{1}$ and A de Silva $^{2}$}

(Index words: Government hospitals, household costs, in-patient care, non-communicable diseases)

\begin{abstract}
Objective To determine the pattern of morbidity and the demographic and socioeconomic characteristics of patients seeking in-patient services for noncommunicable diseases (NCDs) in medical units of a tertiary care hospital, and to estimate the economic burden imposed by these admissions on the households.

Methods A descriptive cross-sectional study was conducted in medical units of the Colombo North Teaching Hospital, Ragama. Data were collected using a pre-tested interviewer-administered questionnaire. Morbidity patterns and demographic and socio-economic
\end{abstract}

characteristics of patients with NCDs were determined. Direct and indirect components of the household cost of hospital stay were estimated.

Results Fifty five per cent of the patients men male and the largest age group (11\%) was 50-54 years. Seventy per cent were above 40 years of age, and $63 \%$ represented social classes 4 and 5 . Diseases of the circulatory system were the commonest (31\%). Median household cost of the total hospital stay was Rs. 852.00 (inter-quartile range Rs. 351.00-1885.00) of which 70\% were direct costs. Median daily cost was Rs. 340.00 (interquartile range Rs.165.00-666.00). Only 44\% of patients

${ }^{1}$ Department of Community and Family Medicine, Faculty of Medicine, University of Kelaniya. ${ }^{2}$ Department of Economics, Faculty of Arts, University of Colombo.

Correspondence: AK, Tel: +94 10112953411 , e-mail: <anuradhini@mfac.kln.ac.lk> (Competing interests: none declared). Received 2 November 2004 and revised version accepted 21 April 2005. 
incurred an indirect cost. Cost of travelling was the main contributor (36\%) to the household cost. Laboratory investigations contributed $16 \%$.

Conclusions Most patients seeking in-patient services were from a poor socioeconomic background. The economic burden imposed by the admission to the household was mainly due to direct costs incurred for travelling and investigations.

\section{Introduction}

The close relationship between health and the economy is increasingly recognised today [1]. Sri Lanka, a country in developmental transition, has achieved high levels of health at low cost [2]. With the increase in life expectancy at birth and reduction in crude birth rate, Sri Lanka's population is ageing rapidly. This has made noncommunicable disease (NCD) an important health problem. Most NCD start in middle age and continue until death, making heavy demands on health care facilities. The individual economic burden of these diseases is borne primarily by households.

About $95 \%$ of the in-patient health care needs of our population are provided by the public sector [3], the facilities ranging from rural hospitals to teaching hospitals with sophisticated facilities. Due to the lack of a systematic referral system, patients bypass small institutions and come to secondary and tertiary care institutions [4].

The purpose of this study was to describe the pattern of NCD morbidity, the sociodemographic and socioeconomic characteristics of patients suffering from NCDs, and to estimate the costs borne by households for in-patient care due to NCDs at a tertiary level public sector health care institution.

\section{Methods}

This cross-sectional study was conducted in the four medical units of the Colombo North Teaching Hospital, Ragama from 24 July to 15 November 2003. Patients admitted with a presumptive diagnosis of an NCD were sampled systematically. The sample of 791 comprised only patients who had a definitive diagnosis of an NCD on discharge (ischaemic heart disease, hypertensive heart disease, endocrine disorders such as diabetes mellitus, cerebrovascular accidents, rheumatoid arthritis, etc). Patients admitted for pre-planned investigations and regular therapeutic procedures, those who left against medical advice or who were transferred to another institution or who died, were excluded. The diseases were classified according to the 10th revision of the International Classification of Diseases [5].

\section{Data collection}

Data were collected using a pre-tested interviewer administered questionnaire by medically qualified investigators after obtaining written informed consent. The subjects were interviewed on a number of occasions during their hospital stay, i.e. soon after admission, every other day during the course of their stay, and on discharge, to update cost data. Where the patient was unable to provide the required information, it was obtained from a reliable informant.

\section{Data analysis}

Data were coded and entered into EpiData 2.1b and analysed using SPSS version 10. Measures of central tendency and variation were used to describe data. The median test [6] was used to compare different groups as the distributions were skewed.

Social class was categorised based on the occupation of the head of the household [7]. A composite socioeconomic score was developed using the occupation and educational level of the head of the household, and some indicators of the accumulated wealth of the family [8].

\section{Ethical considerations}

Ethical committee approval was obtained from the Ethics Committee of the Faculty of Medicine, University of Kelaniya. Permission to conduct the study was obtained from the Director, Colombo North Teaching Hospital, and the Consultant Physicians of the medical units.

\section{Results}

There were $438(55 \%)$ men and the median age of the sample was 50 (inter-quartile range 36-63) years. Sixty six per cent were between $15-59$ years and $31 \%$ were over 60 years. The median family size was four (inter-quartile range 3-5). Eighty two per cent were resident in the Gampaha district with $87 \%$ residing within a radius of $50 \mathrm{~km}$ from the hospital. Five per cent of patients, permanently resident in areas over $100 \mathrm{~km}$ away from the hospital were admitted during temporary visits to the area. About $17 \%$ of household heads had an educational level higher than the G.C.E. (Ordinary Level). Seventy three per cent of households had a monthly family income of less than Rs. 10000.00 per month and $26(3 \%)$ patients had health insurance.

The median duration of hospital stay was 2 days (inter-quartile range1-5). Diseases of the circulatory system were the commonest, including ischaemic heart disease $(55 \%)$, hypertensive disease $(25 \%)$ and cerebrovascular disease $(17 \%)$. Alcoholic liver disease accounted for $48 \%$ of diseases of the digestive system, and asthma accounted for $95 \%$ of diseases of the respiratory system. Among endocrine, metabolic and nutritional diseases, diabetes mellitus was the commonest (96\%). Injury, poisoning and other consequences of external causes (ICD S00-T98) and external causes of morbidity and mortality (ICD-V01-Y98) accounted for $18 \%$ of all medical admissions due to NCDs.

Direct household costs included the costs of travelling, meals, pharmaceuticals, laboratory investigations done in the private sector, patient care, consumable items and 
other miscellaneous costs. Indirect household costs included the costs of lost earnings by the patient and the family. The median direct household cost for the total hospital stay was Rs. 542.00 (inter-quartile range Rs. 208.00-1332.00), the major component being for travelling (Table 3). Over 50\% of patients did not incur any costs for carers, meals, pharmaceuticals and consumables. In $52 \%$ of patients, costs incurred were only for travelling. Less than $5 \%$ of patients had a paid bystander. Forty eight per cent and $34 \%$ of patients incurred costs for meals and pharmaceuticals. Thirty four per cent of patients got laboratory investigations done in the private sector. Consumable items were obtained by $15 \%$ of patients.
Forty four per cent of patients incurred an indirect cost, and the median cost for their entire stay was Rs. 600.00 (inter-quartile range Rs.300.00-1200.00) (Table 4). The median number of working days lost by the patient and family members was 2 (inter-quartile range 1-4 days).

The median total household cost for the entire stay was Rs. 852.00 (inter-quartile range Rs. 351.00-1885.00), of which, direct costs accounted for $70 \%$ (Table 4 ). This is $22 \%$ of the median daily monthly income of an average Sri Lankan for the period 1996/97 [9]. The median cost was Rs. 340.00 (inter-quartile range Rs.165.00-666.00). The total household costs of patients categorised in the higher social classes were significantly higher than that of the lower social classes $(\mathrm{p}=0.032)$.

Table 2. Distribution of diseases according to the ICD 10 classification

\begin{tabular}{lcc} 
Disease Category & Frequency & $(\%)$ \\
\hline Malignant neoplasms (C00-C99) & 14 & $(1.8)$ \\
Diseases of the blood and blood-forming organs and certain disorders & & $(0.8)$ \\
involving the immune mechanism (D50-D89) & 6 \\
Endocrine metabolic and nutritional diseases (E00-E90) & 46 & $(5.8)$ \\
Mental and behavioural disorders (F00-F99) & 18 & $(2.3)$ \\
Diseases of the nervous system (G00-G99) & 41 & $(5.2)$ \\
Diseases of the circulatory system (I00-I99) & 243 & $(30.7)$ \\
Diseases of the respiratory system (J00-J99) & 57 & $(7.2)$ \\
Diseases of the digestive system (K00-K93) & 143 & $(18.1)$ \\
Diseases of the musculoskeletal system and connective tissue (M00-M99) & 60 & $(7.6)$ \\
Diseases of the genitourinary system (N00-N99) & 20 & $(2.5)$ \\
Injury, poisoning and certain other consequences of external causes (S00-T98) & 55 & $(6.9)$ \\
External causes of morbidity and mortality (V01-Y98) & 88 & $(11.1)$ \\
Total & $\mathbf{7 9 1}$
\end{tabular}

Table 3. Direct household costs*

\begin{tabular}{lccc}
\hline Item & Number(\%) incurring cost & Median cost (Rs.) & Inter-quartile range (Rs.) \\
\hline Travelling & $779(98.5)$ & 313.00 & $130.00-670.000$ \\
Bystander & $217(27.4)$ & 164.00 & $67.00-581.00$ \\
Meals & $379(47.9)$ & 70.00 & $40.00-150.00$ \\
Pharmaceuticals & $267(33.5)$ & 150.00 & $72.00-256.00$ \\
Investigations & $265(33.5)$ & 430.00 & $180.00-830.00$ \\
Consumables/other costs & $119(15.0)$ & 82.00 & $30.00-180.00$ \\
\hline
\end{tabular}

*Based on those who incurred a particular cost

Table 4. Components of total household costs

\begin{tabular}{lrrr}
\hline Component & Mean & SD & Percentage \\
\hline Direct cost & 1051.00 & 1598.20 & 69.5 \\
$\quad$ Travelling & 538.70 & 761.40 & 35.6 \\
Bystander & 111.10 & 328.30 & 7.3 \\
Meals & 62.50 & 138.50 & 4.1 \\
Pharmaceuticals & 73.60 & 180.70 & 4.9 \\
Investigations & 244.50 & 833.60 & 16.2 \\
Consumables and other costs & 20.60 & 108.70 & 1.4 \\
Indirect cost & 461.36 & 1271.84 & 30.5 \\
Total cost & $\mathbf{1 5 1 2 . 3 6}$ & $\mathbf{2 2 4 5 . 7 6}$ & $\mathbf{1 0 0 . 0}$ \\
\hline
\end{tabular}




\section{Discussion}

Economic and social changes occurring in developing countries are associated with changes in the morbidity pattern due to the emergence of NCDs. The burden of noncommunicable diseases is felt to a greater extent in low and middle-income economies than in high-income economies [10]. Investments in our health sector are limited, and funding is dwindling gradually due to shrinking state revenue and competing demands. Careful consideration of the pattern of public sector health care utilisation, the socioeconomic level of the clients and patterns of morbidity and mortality are essential.

Prevalence of NCDs increases with advancing age. Seventy per cent of the patients of our sample were over 40 years. The $66 \%$ in the $15-59$ year age group are economically active and cause, a large economic burden as indirect costs due to lost earnings. The male preponderance in this study, probably due to their increased physiological and lifestyle related risk of NCD, leads to a profound economic impact in households.

The majority of patients in this study were from middle and low income households as reflected in the social class and socioeconomic level (Table 1), probably due to the study setting which was a public sector establishment that provides free health care.

Travel costs comprised the major component (36\%) of household costs. Wide variations in travel costs were due to different distances to the hospital and mode of transport used. Costs of provision of meals $(4 \%)$ and engaging carers $(7 \%)$ were less important. As expected, social class was associated with costs of hospitalisation, with patients of higher social classes using private vehicles for transport as compared to those from the poorer social classes who depended on public transport.

Despite the operation of a free health care service, $34 \%$ of patients got some laboratory investigations done in the private sector. Pharmaceuticals were purchased from the private sector by $34 \%$ of patients. With overcrowding in tertiary care settings, pharmaceutical and laboratory services are generally over-whelmed. In addition, certain investigations cannot be performed in state sector hospitals. As lack of finances restricts poor patients from accessing the private sector, outsourcing of these services should be considered, at least on the basis of need.

The costs incurred by patients and families for the entire hospital stay comprised about one quarter of the monthly income of an average Sri Lankan family. The daily cost exceeded the national median daily income of an adult. As chronic diseases recur, the recurrent economic burden can be substantial in low-income groups who have many other economic priorities.

Hospitalised patients represent the severe forms of morbidity, reflecting only the "tip of the iceberg" of the burden of disease in the community. Considering the hidden burden of non-communicable diseases, both inpatient and out-patient facilities required for long term
Table1. Demographic details of patients

\begin{tabular}{|c|c|c|}
\hline Variable & Number & $(\%)$ \\
\hline \multicolumn{3}{|l|}{ Age } \\
\hline$<15$ & 22 & $(2.8)$ \\
\hline $15-24$ & 98 & $(12.4)$ \\
\hline $25-59$ & 425 & $(53.7)$ \\
\hline$>60$ & 246 & (31.1) \\
\hline \multicolumn{3}{|l|}{ Sex } \\
\hline Male & 438 & $(55.4)$ \\
\hline Female & 353 & $(44.6)$ \\
\hline \multicolumn{3}{|c|}{ Area of residence } \\
\hline$<10 \mathrm{~km}$ & 393 & $(49.7)$ \\
\hline $10-49 \mathrm{~km}$ & 299 & (37.7) \\
\hline $50-99 \mathrm{~km}$ & 59 & $(7.5)$ \\
\hline$>100 \mathrm{~km}$ & 40 & $(5.1)$ \\
\hline \multicolumn{3}{|l|}{ Social class ${ }^{\mathrm{a}}$} \\
\hline 1 & 16 & $(2.0)$ \\
\hline 2 & 141 & $(17.8)$ \\
\hline 3 & 140 & $(17.7)$ \\
\hline 4 & 235 & $(29.8)$ \\
\hline 5 & 259 & $(32.7)$ \\
\hline \multicolumn{3}{|c|}{ Socio-economic status ${ }^{\mathrm{b}}$} \\
\hline 1 & 2 & $(0.3)$ \\
\hline 2 & 264 & (33.4) \\
\hline 3 & 278 & $(35.1)$ \\
\hline 4 & 175 & $(22.1)$ \\
\hline 5 & 72 & $(9.1)$ \\
\hline
\end{tabular}

a Social Class

1- Higher professionals and administrative occupations

2- Lesser professionals, industry, retail trades

3- Skilled occupations (manual and non-manual)

4- Semi-skilled occupations

5- Unskilled occupations

${ }^{\mathrm{b} S}$ Socioeconomic status

$$
\begin{aligned}
& \text { 1- Low } \\
& \text { 2- Moderately low } \\
& \text { 3- Moderate } \\
& \text { 4- Moderately high } \\
& \text { 5- High }
\end{aligned}
$$

management of these conditions need to be upgraded in the face of the epidemiological and demographic transition. Improvement of out-patient and follow up facilities will reduce the cost of in-patient care on both households and the health system by minimising the number of hospital admissions.

\section{Acknowledgements}

We acknowledge the help given by Dr. SM Samarage of the Ministry of Health, and Dr. PL Jayawardena, Dr. A Pathmeswaran, Prof. HJ de Silva and Prof. NR de Silva, of the Faculty of Medicine, University of Kelaniya.

We also thank Director and Consultant Physicians of Colombo North Teaching Hospital, Ragama and Drs. MRF Zahriya, RAKK Ranathunge, OMS Weerasinghe and UKT Vipulanayake for their assistance in data collection. 


\section{References}

1. World Health Organisation. Macroeconomics and health: investing in health for economic development. Geneva: Report of the commission on Macroeconomics and Health, 2001.

2. Musgrove P, Zeramdini R, Carrin G. Basic patterns in national health expenditure. Bulletin of the World Health Organization 2002; 80:134-42.

3. Ministry of Health Nutrition and Welfare. Annual Health Bulletin. Colombo, 2001.

4. Ministry of Health, Nutrition and Welfare and Institute of Policy Studies. Sri Lanka National Health Accounts: Sri Lanka National Health Expenditures. Colombo, 2002.
5. World Health Organisation. International Statistical Classification of Diseases and Related Health Problems. 10th revision Vol.1. Geneva, 1992.

6. SPSS for Windows, Release 10.0.1, SPSS Inc, USA. 1999.

7. Barker DJP, Rose G. Epidemiology in Medical Practice. 4th ed. Edinburgh: Churchill Livingstone, 1990.

8. de Silva NR. Intestinal Parasites of the Under-fives in the Mahaiyyawa Slums. M.D. (Medical Microbiology) thesis. Postgraduate Institute of Medicine. Colombo, 1994.

9. Central Bank of Sri Lanka. Consumer Finance and Socioeconomic Survey 1996/97. Colombo. 1999.

10. Gwatkin D, Guillot M, Heuveline P. The burden of disease among the global poor. Lancet. 1999; 354: 586-589. 